\title{
Uterus Present
}

National Cancer Institute

\section{Source}

National Cancer Institute. Uterus Present. NCI Thesaurus. Code C138959.

An indication that an individual's uterus is present. 